\title{
ASSESSING THE CASCADING EFFECT OF ENERGY AND TRANSPORT CRITICAL INFRASTRUCTURE ELEMENTS: CASE STUDY
}

This article focuses on the issue of assessing the cascading effects of critical energy and transport infrastructure elements at the fundamental level. The introductory part deals with the typology of failures and their impacts, which spread through the critical infrastructure system. At this stage, the paper presents current approaches to assessing the cascading effects and, in particular, addresses a newly developed assessment methodology. The following part defines the initial conditions of assessment and describes selected elements from the areas of energy and rail transport to which the methodology will be subsequently applied. The main part of the article is a case study of the proposed methodology, assessing the cascading effects by calculating the value of their risks, depending on the resilience and correlation of the rated elements.

Keywords: critical infrastructure, cascading effect, assessing, resilience, correlation

\section{Introduction}

Infrastructure failures brought about by system malfunction or disruption due to a terrorist attack or natural or technical causes are likely to increase significantly the extent of impacts on the failure of other dependent infrastructure. This is due to interdependencies existing between infrastructure segments which can exert a direct influence on these effects. In their paper, Zimmerman and Restrepo [1] presented the two steps aimed at facilitating the understanding of interdependencies. First, the likelihood of interdependencies between infrastructures is determined. Any identified interdependencies are subsequently categorized according to their location. A basic overview of the approaches to perceiving correlations constitutes the primary aspect affecting the identification and understanding of interdependencies. Rinaldi et al. [2] also identified the environment in which, and the degree to which, infrastructures are connected or interconnected. The authors argue that the types of interdependencies are of considerable relevance.

With respect to critical infrastructure, one should not fail to appreciate the basic difference between dependency and interdependency [3]. The critical infrastructure dependency refers to the link between two infrastructures where the condition of one affects the condition of the other. Interdependency, as opposed to dependency, implies a mutual relationship between two or more infrastructures. Those dependencies may be classified into several types. Buhne et al. [4], for example, divides dependencies into Requires-dependency, Exclusive-dependency, Hints-dependency and Hinders dependency. Hromada et al. [5] emphasize the need to make a clear distinction between positive and negative linkages. According to Rinaldi et al. [2], interdependency can be further classified as: physical, cyber, geographic and logical.

Infrastructure dependencies determine individual types of failures or effects [2]. Basic failures include a cascading failure, which occurs when a disruption in one infrastructure causes the failure of a component in the second infrastructure, which in turn leads to a disruption in the second infrastructure [6]. An escalating failure occurs when an existing disruption in one infrastructure exacerbates an independent disruption in the second infrastructure. A common-cause failure occurs when two or more infrastructure networks are disrupted at the same time.

\section{Approaches to assessing cascading effects}

There is currently a multitude of approaches to assessing cascading effects in critical infrastructures. The majority of these go hand in hand with the issue of evaluating the resilience

\footnotetext{
* 'Veronika Brabcova, ${ }^{1}$ Simona Slivkova, ${ }^{1}$ David Rehak, ${ }^{2}$ Fulvio Toseroni, ${ }^{3}$ Jan Havko

${ }^{1}$ Faculty of Safety Engineering, VSB - Technical University of Ostrava, Czech Republic

${ }^{2}$ Department of Life and Environmental Sciences, Universita Politecnica delle Marche, Italy

${ }^{3}$ Faculty of Security Engineering, University of Zilina, Slovakia

E-mail: david.rehak@vsb.cz
} 


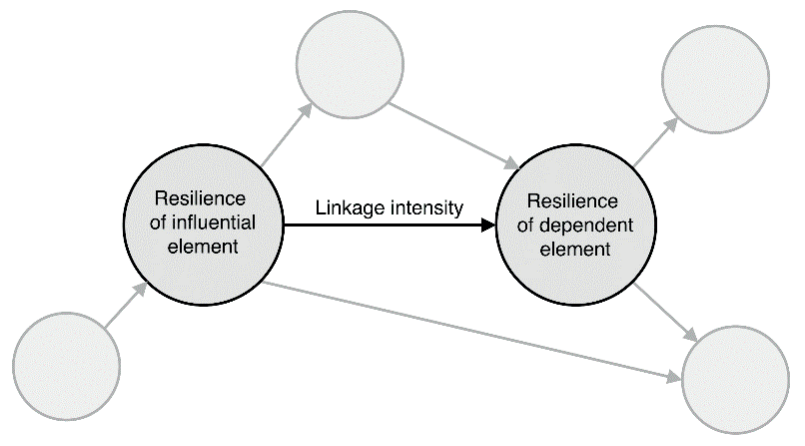

Figure 1 Relationship between variables for calculating the risk of cascading effect propagation

of a given system. Omer et al. [7] looked into a methodology, which proposes the application of network analysis. Via graph theory, they were able to identify the critical passage and thus establish the critical nodes with the most severe impacts on a selected network. This is well complemented by Hromada et al. [5], who developed a maths-based methodology and, in doing so, formalized a general approach to evaluating the resilience of selected critical infrastructure elements and networks. By contrast, Muller [8] advocated an approach to evaluating the resilience of an entire system via the fuzzy logic.

Aung and Watanabe [9], Dudenhoeffer et al. [10], Markuci and Rehak [11] and Rehak et al. [12] were among those to explore in greater detail the issue of modelling the interdependencies between individual infrastructures. All models can essentially be said to deal with the establishment of resilience indicators, which may vary slightly in individual publications. They are all aimed at evaluating critical infrastructure resilience or the linkages between individual critical infrastructure sectors.

A new approach to assessing the cascading effects in critical infrastructure systems was put forward in research projects Nos. VI20152019049 “'RESILIENCE 2015: Dynamic Resilience Evaluation of Interrelated Critical Infrastructure Subsystems" and SP2017/87 "Assessing the Correlation of Selected Sectors in a Critical Infrastructure System." The completed research led to establishment of a procedure for assessment of the cascading impacts, involving assessment of the level of risk of impacts spreading between critical infrastructure elements. That approach hinges on the ability to evaluate the resilience of influential and dependent elements (the degree of the transferred impacts is assessed based on these variables) and the intensity of the linkage between those elements (the linkage intensity determines the likelihood of impact transfer). See Figure 1 for the relationship between variables for calculating the risk of cascading effect propagation.

Based on the above, the risk of cascading impact propagation can be calculated using the following Equation:

$$
R=I \cdot P=V_{I E} \cdot V_{D E} \cdot L I=\left(1-R E_{I E}\right) \cdot\left(1-R E_{D E}\right) \cdot L
$$

where $R$ is the risk level of a cascading effect spreading from an influential element (IE) onto a dependent element (DE) [\%]; $I$ is the degree of transferred impacts [\%]; $P$ is the probability of impact transfer [\%]; $V_{I E}$ is influential element vulnerability [\%]; $V_{D E}$ is dependent element vulnerability [\%]; $L I$ is the linkage intensity between influential and dependent elements [\%]; $R E_{I E}$ is the resilience of influential element [\%]; $R E_{D E}$ is resilience of dependent element [\%].

Element resilience is established based on the following Equation:

$R E=\frac{\sum_{i}^{n} C_{R E}}{n}=\frac{\sum_{i}^{n}\left(\frac{\sum_{i}^{m} P_{C}}{m} \cdot \frac{100}{P_{C_{\max }}}\right)}{n}$

where $R E$ is the resilience level of a critical infrastructure element [\%]; $C_{R E}$ is the evaluated level of components determining the resilience of a critical infrastructure element [\%]; $n$ is the number of evaluated components of element resilience; $P_{C}$ is the evaluated level of parameters determining the components of element resilience [a numerical value on a scale of 1 (i.e. the worst possible rating) to 5 (i.e. the best possible rating)]; $m$ is the number of evaluated parameters [13].

The intensity of linkages between elements is determined based on the weighted average using the following Equation:

$L I=\frac{\sum_{i}^{p} P_{L I} \cdot w}{\sum_{i}^{p} w} \cdot \frac{100}{P_{L L_{\max }}}$

where $L I$ is the linkage intensity between influential and dependent elements [\%]; $P_{L I}$ is the evaluated level of parameters determining the element linkage intensity [a numerical value on a scale of 1 (i.e. the worst possible rating) to 3 (i.e. the best possible rating)]; $w$ is the weights of parameters determining the element linkage intensity.

\section{Initial conditions of the case study}

A case study assessing the risk of cascading impact propagation between the critical infrastructure elements was carried out with a view to presenting the newly developed methodology. Two elements were selected for practical demonstration purposes: a transformer station (i.e. an influential energy sector element) and a level-crossing warning system (i.e. a dependent railwaytransport sector element).

Power stations are located at power system nodes and are designed to transform, distribute, convert or offset electrical energy. For the purposes of the present study, the transformer station designed for the conversion of electrical energy to the required voltage and its subsequent distribution was selected. It employs power transformers interconnecting two or more networks with various voltages. Its layout is determined by the design of individual distribution facilities or substations and the placement of power transformers [14]. Pursuant to the Energy 


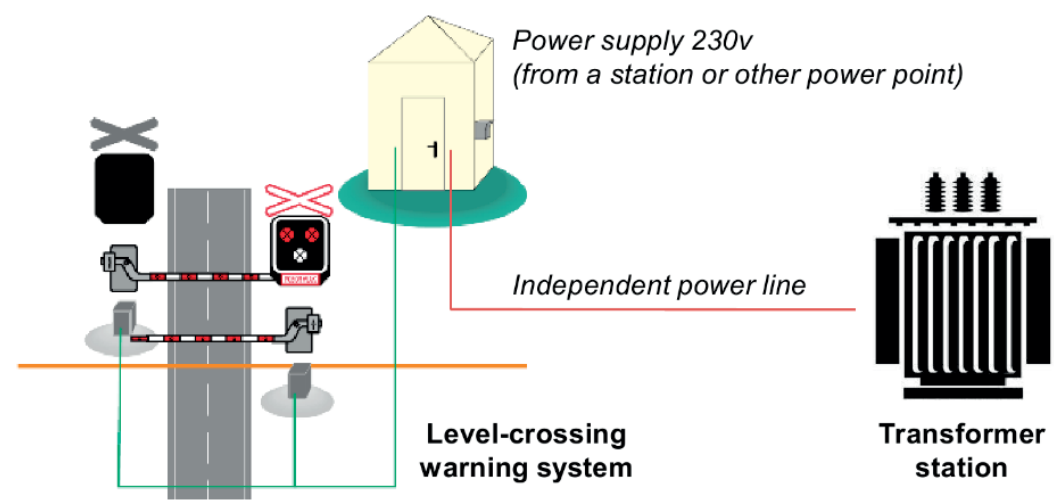

Figure 2 Practical link diagram of the influential and dependent elements

Act [15], the operator of the distribution system is obliged to ensure its reliable operation, modernization and development the failure to provide electricity may be contractually penalized.

A level-crossing warning system is designed to improve safety at intersections where single or multiple railway lines cross a road at the same level. Level-crossing warning systems must have a guaranteed power supply conforming to Category 1 , as stipulated in CSN 376605 [16]. This category includes systems whose failure due to power outage might pose an immediate threat to human life or lead to major property damage. Evidence of such impacts may be provided by an assessment of the risks in critical railway infrastructure [17]. The linkage between the two elements is illustrated in Figure 2.

In order to effectively evaluate cascading impacts in a critical infrastructure system, the scenarios of emergency impacts on the elements being evaluated must be viewed within the context of specific threats, which can be classified in five basic groups [18]:

- climatological (i.e. natural disasters such as floods, hurricanes, heavy snowfall);

- geological (e.g. earthquakes, volcanic activity, landslides);

- biological (i.e. epidemics, pandemics, epiphytic or epizootic diseases);

- technological (i.e. technological emergencies such as radiation emergencies, hazardous chemical spills, flooding caused by damage to hydraulic structures, widespread disruptions to engineering networks or public water supply emergencies);

- $\quad$ social (i.e. terrorism, criminal activity).

This particular case study involves a blackout caused by a terrorist attack, which is statistically one of the most common criminal threats [19].

\section{Evaluating resilience of selected elements}

Resilience is one of the key factors contributing to the preservation of critical infrastructure element functionality. It represents the ability of elements to mitigate the intensity of impacts caused by an emergency event and reduce the duration of their failure or disruption. The required level of resilience can be achieved via continuous enhancement of the five basic areas: preparedness, absorption, responsiveness, recoverability and adaptability. These areas and their criteria (Figure 3) determine the level of resilience of critical infrastructure elements and thereby markedly decrease their vulnerability [20].

The following evaluation of the resilience of both elements is carried out in the context of the selected emergency, i.e. a blackout caused by an act of terrorism.

\subsection{Preparedness}

Preparedness of critical infrastructure elements revolves around activities which help to oppose the effects of emergencies; this entails planning and having the required measures, forces and means in place to successfully manage any emergency and recover from its impacts [20]. Preparedness is determined by three parameters: risk analysis (1-5), planning (1-5) and implementation of measures (1-5).

The distribution system operator relies on preparedness in planning to cope with emergencies. At the same time, the organization adopts, as part of its continuous improvement policy, new measures aimed at enhancing its effectiveness in responding to emergencies. In consideration of this, the level of preparedness of the transformer station with regard to the aforementioned parameters was rated as " 4 ".

The risk analysis of the railway system element operator focused on risks associated with the warning system in relation to emergencies that are common in the railway transport sector. Based on this analysis, the operator proceeded to draw up individual safety/security plans. Some plans necessitated the adoption of mitigating measures designed to alleviate the impacts of emergencies on the railway transport system. Having analysed this information, all of the three parameters were rated as " 3 ". 


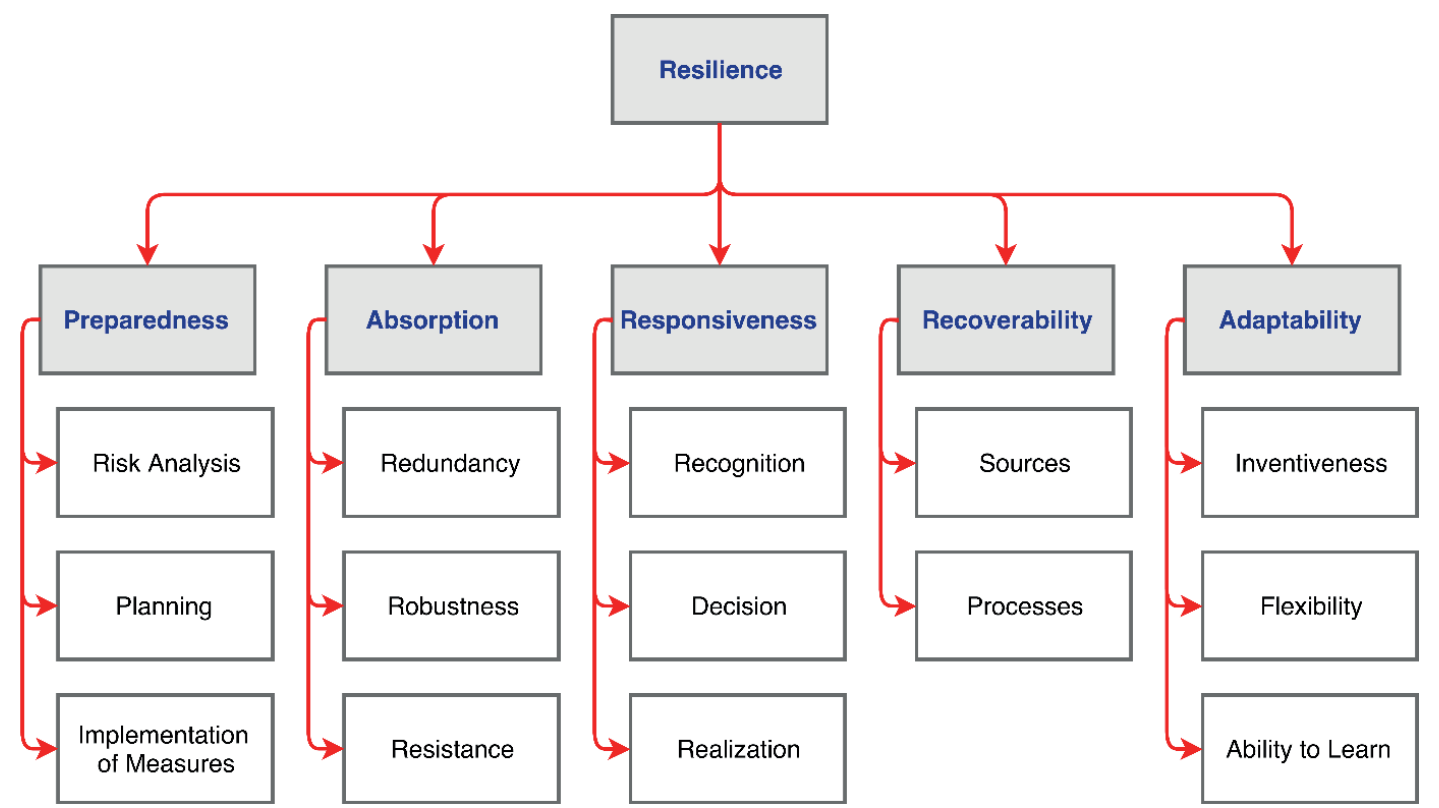

Figure 3 Areas and their criteria determining the resilience of critical infrastructure elements

\subsection{Absorption}

Absorption is the internal property of an element, which expresses its ability to automatically absorb the impacts of system failures and thereby to minimize their consequences [20]. It is one of the basic properties of an element prior to an emergency, which further determines to what extent the element will have to be recovered. Absorption enables the element to withstand negative events without any substantial deviation from the required function. Absorption is determined by three parameters: redundancy (1-5), robustness (1-5) and resistance (1-5).

With regard to redundancy, the transformer station was rated as " 3 ", because despite it being a branched network where different loops can be activated during a failure, this option is not always available. The robustness of the transformer station was rated as " 1 ". However, its resistance, i.e. its capacity to learn from previous events, was rated as " 3 ".

The level-crossing warning system has a significant redundant source (i.e. a backup power source for up to 8 hours), which makes it possible to rate the redundancy of the dependent element as "4". However, neither the robustness nor the resistance of this element are considered as substantial. As this particular element is not supported or protected in any specific manner, both parameters were rated as " 3 ".

\subsection{Responsiveness}

Responsiveness can be understood as the ability of an element to react to an emergency promptly and efficiently in order to minimize its impacts on the function or existence of the element [20]. Responsiveness is determined by three parameters: the time it takes to recognize an emergency (1-5), the time it takes to adopt a solution (1-5) and the time it takes to respond (1-5).

The technical design of the control room and the considerable knowledge of its operators substantially contribute to the prompt recognition of failures and facilitate the effective response of the distribution system operator. In the case of the transformer station, all three parameters were thus rated as " 5 ".

With respect to the level-crossing warning system, the time it takes to recognize an emergency was rated as "4" due to its linkage to other systems (e.g. a control room). As the adoption and response parameters may to a considerable extent vary from one control room operator to another, they were rated as " 3 ".

\subsection{Recoverability}

Recoverability expresses the time period of recovery, i.e. the time necessary for the recovery of function of the element to the required level after its disturbance by an emergency [20]. Recoverability is determined by the two parameters: allocated sources of recovery, i.e. human, material, financial and informational (1-5) and recovery processes (1-5).

Considering the penalties stipulated by the Energy Act [15] for failing to ensure the reliable operation, modernization and development of the distribution system, and considering the substantial losses resulting from the failure to provide contractual services, the allocated sources were rated as " 4 ". The recovery processes parameter was rated as " 5 " due to the legal obligation of the distribution system operator to ensure its function. 

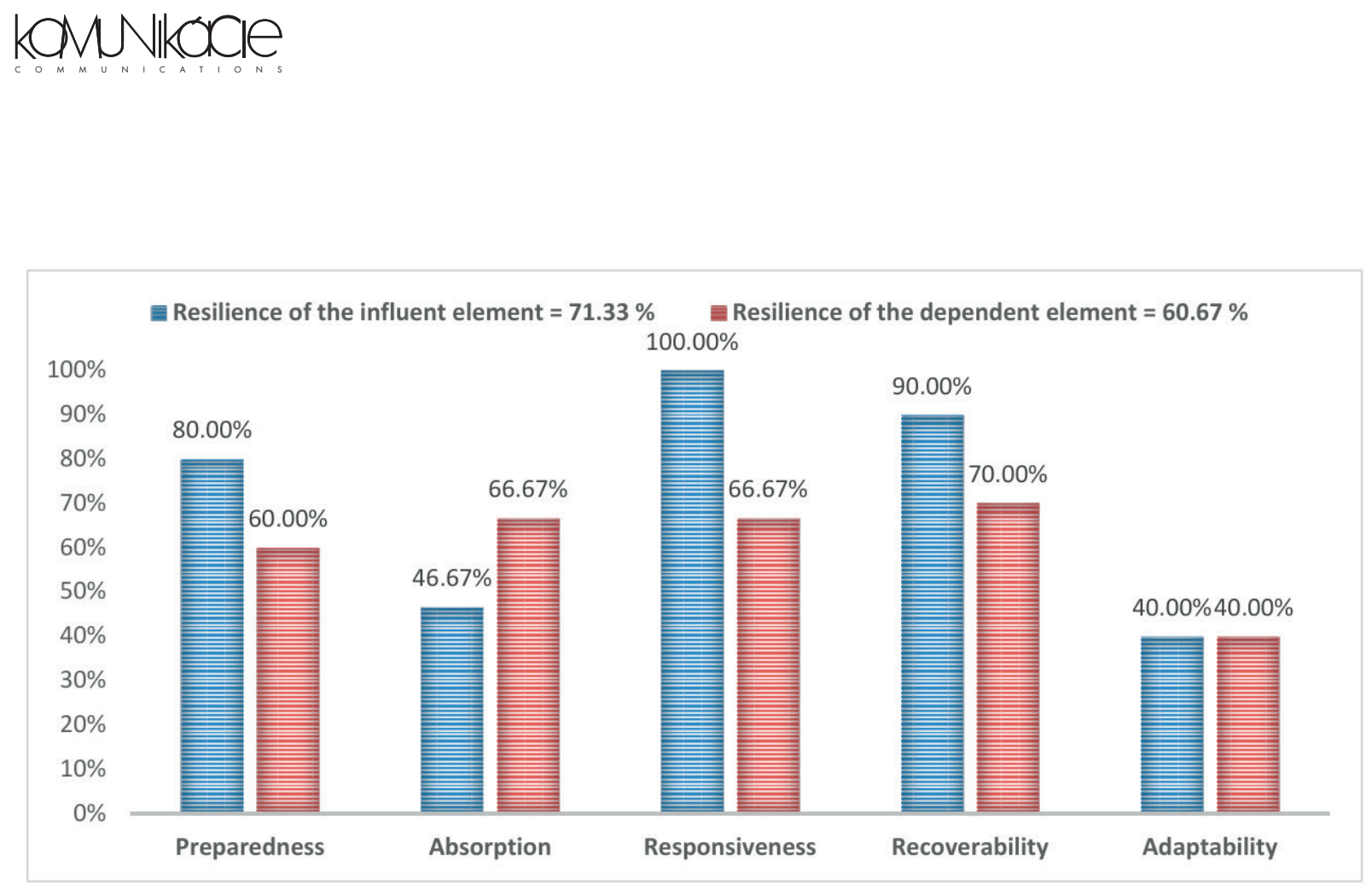

Figure 4 The level of influential and dependent element resilience

The allocated sources parameter related to the level-crossing warning system was rated as "2" due to the limited availability of some of the required sources. With respect to the dependent element recovery process following the complete recovery of the influential element, an immediate recovery of function may be expected; that is why this parameter was given the full rating of " 5 ".

\subsection{Adaptability}

Adaptability is the ability of an organization to adapt its element to the effects of an emergency. It represents the dynamic (long-term) ability of an organization to adapt to changes in circumstances [20]. Adaptability is determined by three parameters: the level of inventiveness (1-5), the level of flexibility (1-5) and the ability to learn (1-5).

The distribution system operator is fairly limited in its ability to adapt the actual transformer station to new conditions. However, the operator may adaptively support its overall activity through research, modernization and other development activities, and thereby apply any new findings to the end elements of its system, as well. Accordingly, the parameters of the level of inventiveness and flexibility and the ability to learn were all rated as "2".

The structural and technological design of the level-crossing does not allow for much adaptability. The operator of the element may partially address these aspects through its activity, especially in cases where new options are explored with a view to managing emergencies that have previously affected its area of operation. With respect to the level-crossing warning system, all of the three parameters defined above were given the rating of " 2 ".

\subsection{Resilience}

After the above-mentioned values of individual parameters have been entered into Equation (2), the level of resilience of the two elements being evaluated can be determined (see Figure 4).

\section{Evaluating correlation of selected elements}

An important factor affecting the correlation between the critical infrastructure elements, is the linkage intensity, which determines the speed and manner in which impacts can spread between individual elements [21]. If the intensity is low, even less resilient elements can be protected. The linkage intensity between influential and dependent critical infrastructure elements is determined by six basic parameters: the type of linkage (1-3), the condition of linkage (1-3), the level of linkage (1-3), the time characteristics of linkage (1-3), the substitution of linkage (1-3) and the structure of linkage (1-3). Due to their varying levels of significance, the parameters were assigned the following weighting coefficients: the type of linkage (0.23), the condition of linkage (0.23), the level of linkage (0.17), the time characteristics of linkage (0.15), the substitution of linkage (0.12) and the structure of linkage (0.10). The weighting coefficients for linkage intensity were determined in terms of grant project SP2017/87 "Assessing the Correlation of Selected Sectors in a Critical Infrastructure System".

In evaluating the type of linkage between the transformer station and the level-crossing warning system, physical interconnectedness was found to exist between the two elements and the parameter was accordingly rated as " 3 ". 
Resilience of influential element $R E_{I E=} 71.33 \%$

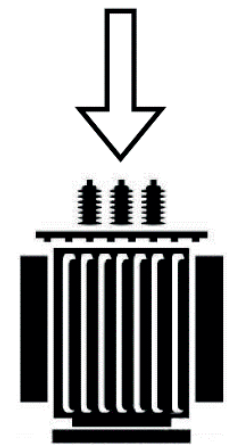

Transformation Station
Resilience of dependent element $R E_{D E}=60.67 \%$

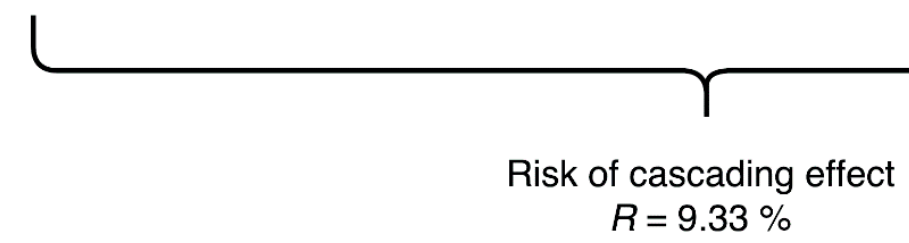

Figure 5 The risk of impact propagation between influential and dependent critical infrastructure elements

The function of the level-crossing warning system is dependent on the power supply, and even though it has a backup power source to rely on in the event of a blackout, its capacity is limited to 8 hours of operation only. For this reason, the dependency option was factored into evaluating the condition of the linkage and the parameter was given the rating of " 2 ".

Operation of the level-crossing warning system is completely dependent on the power supply, thus constituting an intra-sectoral linkage. Consequently, the level of linkage was rated as "2".

Since the serviceability of the level-crossing warning system hinges on the continuous supply of electricity, the linkage was rated as " 3 " in terms of its time characteristics.

In the event of disruptions in the power supply, it is possible to activate different network loops in order to maintain the power supply to the equipment for the duration of the repair work. For this reason, the existence of one substitution linkage with a rating value of " 2 " was selected to evaluate linkage substitution between individual elements.

As the linkage between the transformer station and the levelcrossing warning system is direct and does not penetrate any node, the linkage structure was given the rating of " 3 ".

After the above-mentioned values of individual parameters have been entered into Equation (3), the determined linkage intensity between the influential and dependent elements was found to have an $82.7 \%$ probability of impact transfer.

\section{Evaluating the risk of impact propagation between selected elements}

The evaluation of cascading effects in a critical infrastructure system consists of determining the level of risk of impact propagation between individual critical infrastructure elements. This essentially involves evaluation of the level of resilience of influential and dependent elements (determining the magnitude of impacts) and the linkage intensity between these elements (determining the likelihood of impact propagation).

After the above-mentioned values, relating to the resilience of the influential and dependent elements and the intensity of their linkage, have been entered into Equation (1), the level of risk of impact propagation between these elements can be determined. The resulting value assigned to the risk of impact propagation between the selected elements was $9.33 \%$ (see Figure 5).

\section{Conclusion}

The continuous progress in scientific and technical knowledge inevitably leads to ever-increasing demands placed on critical infrastructure. Its structure makes it a composite system containing a vast array of elements and linkages. The disruption or failure of some elements may result in the transfer of impacts to other elements and, eventually, in fatal consequences for society, i.e. the loss of human life. That is why the ability to predict the propagation of impacts or cascading effects in a critical 


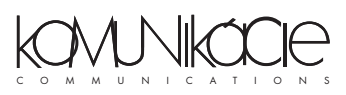

infrastructure system is an important preventive tool, ensuring timely prediction of weak/vulnerable areas and implementation of adequate safety/security measures.

Based on this, the authors of the article developed a methodology for the correlations assessment in the critical infrastructure system. This methodology is based on evaluating the level of interest elements resilience and the link intensity between them. The result of the assessment is to determine the risk level percentage of the cascade effect spreading from the influent element to the dependent element. The methodology is applicable to all technical sectors of critical infrastructure, i.e. energy, communications and information technologies, transport and water management.

\section{Acknowledgement}

This research was supported by the Ministry of the Interior of the Czech Republic under Project VI20152019049 “RESILIENCE 2015: Dynamic Resilience Evaluation of Interrelated Critical Infrastructure Subsystems"and by the VSB - Technical university of Ostrava under Project SP2017/87 "Assessing the Correlation of Selected Sectors in a Critical Infrastructure System”.

\section{References}

[1] ZIMMERMAN, R., RESTREPO, C. E.: Analyzing Cascading Effects within Infrastructure Sectors for Consequence Reduction. Proceedings of IEEE Conference on Technologies for Homeland Security (HST'09), USA, 165-170, 2009. DOI: 10.1109/ THS.2009.5168029

[2] RINALDI, S. M., PEERENBOOM, J. P., KELly, T. K.: Identifying, Understanding and Analyzing Critical Infrastructure Dependencies. IEEE Control Systems Magazine, 21(6), 11-25, 2001. DOI: 10.1109/37.969131

[3] REHAK, D., NOVOTNY, P.: Bases for Modelling the Impacts of the Critical Infrastructure Failure. Chemical Engineering Transaction, 53, 91-96, 2016.

[4] BUHNE, S., HALMANS, G., POHL, K.: Modelling Dependencies between Variation Points in Use Case Diagrams. International Workshop on Requirements Engineering: Foundation for Software Quality (REFSQ'03), Germany, 59-69, 2003.

[5] HROMADA, M., LUKAS, L., MATEJDES, M., VALOUCH, J., NECESAL, L., RICHTER, R., KOVARIK, F.: System and Method of Assessing Critical Infrastructure Resilience. Association of Fire and Safety Engineering, Ostrava, p. 177, 2013.

[6] KROCOVA, S., REZAC, M.: Infrastructure Operation Reliability in Built-Up Areas. Communications - Scientific Letters of the University of Zilina, 1(18), 75-78, 2016.

[7] OMER, M., MOSTASHARI, A., LINDEMANN, U.: Resilience Analysis of Soft Infrastructure Systems. Procedia Computer Science, 28, 873-882, 2014. DOI: 10.1016/j.procs.2014.03.104

[8] MULLER, G.: Fuzzy Architecture Assessment for Critical Infrastructure Resilience. Procedia Computer Science, 12, 367-372, 2012. DOI: $10.1016 /$ j.procs.2012.09.086

[9] AUNG, Z. Z., WATANABE, K.: A Framework for Modeling Interdependencies in Japan's Critical Infrastructures. Palmer, CH., Shenoi, S. (Eds.): Critical Infrastructure Protection III, Springer, Hanover, 243-257, 2009.

[10] DUDENHOEFFER, D. D., PERMANN, M. R., MANIC, M.: CIMS: A Framework for Infrastructure Interdependency Modeling and Analysis. IEEE Winter Simulation Conference (WSC'06), USA, 478-485, 2006. DOI: 10.1109/WSC.2006.323119

[11] MARKUCI, J., REHAK, D.: Interdependencies of the Critical Infrastructure. Proceedings of International Conference on Fire Protection, Czech Republic, 207-210, 2014.

[12] REHAK, D., HROMADA, M., RISTVEJ, J.: Indication of Critical Infrastructure Resilience Failure. Cepin, M., Bris, R. (Eds.): Safety and Reliability - Theory and Application (ESREL), CRC Press, London, 963-970, 2017.

[13] LOVECEK, T., SVENTEKOVA, E., MARIS, L., REHAK, D.: Determining the Resilience of Transport Critical Infrastructure Element: Use Case. Proceedings of International Scientific Conference Transport Means, Lithuania, 824-828, 2017.

[14] Transformation Station. Electrotechnical Magazine, [online], 2008. Available: http://www.etm.cz/index.php/etm/starsi/38energetika/139-transformacni-stanice.

[15] Act No. 458/2000 Coll., On the Conditions of Business and the Performance of State Administration in the Energy Sectors and on the Amendment of Some Laws (Energy Act).

[16] SSN 376605 ED.2. Connections of Electrical Equipments of Railway on Electric Power. Czech Office for Standards, Metrology and Testing, Prague, p. 20, 2012. 
[17] TITKO, M., BYRTUSOVÁ, A.: The Risk Assessment of Critical Rail Infrastructure. Proceedings of International Scientific Conference on Transport Means, Lithuania, 99-102, 2015.

[18] REHAK, D., MARTINEK, B., RUZICKOVA, P.: Population Protection in the Context of Current Security Threats. Association of Fire and Safety Engineering, Ostrava, p. 131, 2015.

[19] SEDLACIK, M., ODEHNAL, J., FOLTIN, P.: Classification of Terrorism Risk by Multidimensional Statistical Methods. Proceedings of International Conference on Numerical Analysis and Applied Mathematics (ICNAAM'14), Greece, 1648(1), article no. 720011, 2015. DOI: 10.1063/1.4912948

[20] REHAK, D., SLIVKOVA, S., BRABCOVA, V.: Indication of Critical Infrastructure Resilience Failure. Cepin, M., Bris, R. (Eds.): Safety and Reliability - Theory and Application (ESREL), CRC Press, London, 955-962, 2017.

[21] REHAK, D., SENOVSKY, P., HROMADA, M., LOVECEK, T., DVORAK, Z., RISTVEJ, J., SVENTEKOVA, E., SLIVKOVA, S., NESPOROVA, V., BRABCOVA, V.: Bases for Assessing the Correlation of Critical Infrastructure Subsystems. VSB - Technical University of Ostrava, Ostrava, p. 22, 2016. 